\title{
INFARCTION-NEPHRECTOMY FOR METASTATIC RENAL CARCINOMA
}

\author{
Southwest Oncology Group Study
}

\author{
JAMES E. GOTTESMAN, M.D. \\ E. DAVID CRAWFORD, M.D. \\ H. BARTON GROSSMAN, M.D.
} PETER SCARDINO, M.D. JOSEPH D. MCCRACKEN, M.D.

From the Seattle Tumor Institute of Swedish Hospital Medical Center, Seattle, Washington; University of New Mexico, Los Cruces, New Mexico; University of Michigan Medical Center, Ann Arbor, Michigan; Baylor College of Medicine, Houston, and Brooke Army Medical Center, San Antonio, Texas

\begin{abstract}
Thirty patients with metastatic renal cell cancer were treated by renal infarction, followed by delayed nephrectomy. All cases were collected over an eighteen-month period, with a minimum follow-up of one year. There were no complete remissions and only one partial remission, which lasted twenty-one months before progression of disease. Three patients had stable disease for at least six months, but eventually all patients showed evidence of progression. After tumor progression was documented patients were treated with intramuscular medroxyprogesterone acetate (Depo-Provera) $800 \mathrm{mg}$ per week. No patient responded to this therapy. Overall, a 28 per cent oneyear survival and a seven-month median survival were realized, which is similar to other series in which no therapy or palliative nephrectomy was performed. We conclude that infarction and nephrectomy is not an effective modality in the treatment of metastatic renal cell carcinoma. In addition, medroxyprogesterone was not shown to be significantly active against renal cancer in this study.
\end{abstract}

One fourth to one half of patients with renal cell carcinoma are found to have metastatic disease at the time of initial presentation. ${ }^{1,2}$ Historically, frustration has been the only apt description of the efforts to treat this disease with chemotherapy, radiation therapy, hormonal and immunotherapy. ${ }^{3}$ However, several investigators have suggested that renal infarction followed by delayed nephrectomy, with

\footnotetext{
*This investigation was supported in part by the following PHS Cooperative Agreement grant numbers awarded by the National Cancer Institute, DHHS: CA-20319, CA-12213, CA-27057, CA03392, CA-22411, CA-12014, and CA-32102.
}

concomitant institution of progestin therapy, offered improved survival.4,5 The Southwest Oncology Group, attempting to corroborate this information, studied 31 consecutive patients with metastatic renal cell carcinoma, using infarction-nephrectomy only as the initial therapeutic modality.

\section{Material and Methods}

Thirty-one eligible patients were registered between January, 1980, and July, 1981, with a minimal one-year follow-up. Thirty patients were evaluable. Eligible patients were those with disseminated renal cell cancer with a 
measurable lesion, in whom the metastatic disease was not resectable at the time of nephrectomy. All patients had an expected survival of at least three months and were medically capable of tolerating the intended nephrectomy. Exclusions included prior irradiation, chemotherapy, or progestin therapy. Pretreatment studies included intravenous pyelogram, computerized tomography (CT) scan of the abdomen, renal and celiac arteriogram, pulmonary tomograms or CT of the lung, bone and liver scans, and a complete battery of metabolic tests. After eligibility had been determined, the involved kidney was infarcted. The technique has been described previously ${ }^{4}$ and in most instances Gelfoam pledgets were injected directly into the renal artery, followed by placement of Gianturco coils for complete renal artery occlusion. The patients were monitored closely in the postinfarction period, which lasted between six and nine days.

Complete remission was defined as disappearance of all clinically active tumor for a minimum of four weeks. Partial remission was a 50 per cent or greater decrease in the crosssection diameter of the lesion maintained a minimum of four weeks.

When progression of disease was identified, each patient was started on medroxyprogesterone $800 \mathrm{mg}$ intramuscularly per week and followed until disease progression. The patients were then allowed to receive other modalities of treatment, but were followed until death.

\section{Results}

Thirty patients were followed a minimum of one year. One perioperative death occurred. No complete responses and one partial response lasting twenty-one months was achieved. Six patients were alive at the end of the study at twenty-six, twenty-five, fifteen, fifteen, fourteen, and twelve months. Twenty-four patients died within the range of the study. Only $4 \mathrm{pa}$ tients $(13 \%)$, including the partial remission, had stable disease for at least six months after the infarction-nephrectomy. These 4 included 2 males with lung metastases, a female with perihilar nodes, and, the partial remission, a male with chest-wall metastasis. Only 28 per cent of the patients survived one year, and the median survival was seven months. The median time to progression after infarction-nephrectomy was eight weeks. Of eight patients who survived one year, five had evidence of progression before the twelve-month mark. Only 3 of 11 patients with lung-only metastasis lived one year, despite the historic suggestions that these patients were the ones most likely to respond. ${ }^{3}$ Interestingly, 2 patients had evidence of partial remission of lung metastases, while other metastatic deposits grew. All patients eventually showed progression of disease, and those still living are now on other forms of therapy.

Seventeen patients presented with single organ metastases, 11 of these being lung only. Thirteen patients presented with multiple organ metastases. The most common metastatic sites in 30 patients were the lung (21), bone (6), and liver (4).

Pathologic evaluation after nephrectomy revealed capsule invasion in 65 per cent, positive nodes in the perihilar region in 24 per cent, and microscopic venous involvement in 55 per cent. The degree of infarction seen on the pathologic specimen ranged from multifocal to massive, without apparent bearing on the subsequent survival.

The possibility of hyperthermia creating a tumor response was evaluated, as it was common for fever to develop in the postinfarction period. Only 1 of 3 patients in whom significant and sustained fever developed postinfarction was alive at one year. Two of 8 patients who had moderate fever were alive at one year, and 5 of 18 patients with little or no fever were alive for greater than one year.

Patients who exhibited progression after infarction-nephrectomy were prescribed medroxyprogesterone, and 18 patients are evaluable at this time. There were no complete or partial responses, and only 2 of 18 were stable for at least six months. The average time to progression on medroxyprogesterone was 2.5 months. The 2 patients who were stable for six months both died during the study, at twelve and fourteen months after the initial infarction-nephrectomy.

Postinfarction problems were quite rare, although most patients had significant pain. In a few of the patients severe nausea and ileus developed which, in some cases, required nasogastric suction. No mortalities occurred in the postinfarction period, and the only postoperative death occurred in a patient with advanced metastatic liver disease, who died fourteen days postnephrectomy of a cerebrovascular accident. No apparent complications of the arteriogram or embolization itself occurred, although the risk of embolization to the lower extremitics or bowel was a technical possibility. 


\section{Comment}

The proposed mechanism of response to infarction-nephrectomy was thought to represent stimulation of the host immune system secondary to a shower of tumor antigens released into the circulation after the infarction. ${ }^{4}$ Whether this hypothesized stimulation represents a cellmediated response, humoral antibody response, or a reduction of blocking factors is unknown. The end result, however, suggests that the immune response was not significant enough to achieve an active role against the spreading cancer. 'The high response rate of previously reported M.D. Anderson series has apparently not been found in subsequent patients treated by them using similar methods, although they still believe that lung-only metastases fare better with infarction-nephrectomy medroxyprogesterone. ${ }^{6}$ This suggestion was not confirmed in our study since only 3 of 11 patients with lung-only metastasis survived more than one year. Anecdotal cases in the literature of responses to infarction-nephrectomy have been reported, but probably represent the rare cases of spontaneous remission which have been reported in the past with palliative nephrectomy. ${ }^{5}$

The results of renal artery embolization with delayed nephrectomy as sole therapy for metastatic renal cell cancer are not encouraging. In addition, we have not been able to demonstrate activity of medroxyprogesterone against renal cell cancer.

Chemotherapeutic agents that have activity against renal cell cancer need to be developed. To date, hormonal and immune therapy have not demonstrated consistent activity against renal cell cancer, but certainly are no worse than chemotherapy. Research for active agents should continue in all areas of anti-tumor therapy.

1221 Madison \#1210

Seattle, Washington 98104

(DR. GOTTESMAN)

\section{References}

1. Skinner DG, et al: Diagnosis and management of renal carcinoma, Cancer 28: 1165 (1971).

2. Lokch JJ, and Harrison JH: Renal cell carcinoma: natural history and chemotherapeutic experience, J Urol 114: 371 (1975).

3. Skinner DG, and deKernion J: Clinical manifestations and treatment of renal parenchymal tumors, in: Genitourinary Cancer, Philadelphia, WB Saunders Co., 1978.

4. Swanson DA, Wallace S, and Johnson DE: The role of embolization and nephrectomy in the treatment of metastatic renal carcinoma, Urol Clin North Am 7: 719 (1980).

5. Mohr SJ, and Whitesel JA: Spontaneous regression of renal cell carcinoma metastases after preoperative embolization of primary tumor and subsequent nephrectomy, Urology 14: 5 (1979).

6. Swanson DA, et al: Angioinfarction plus nephrectomy for metastatic renal cell carcinoma, an update, J Urol 130: 449 (1983). 